\title{
Use of hearing aids in infancy
}

Congenital sensorineural hearing loss often remains undetected until the second or third year of a child's life. ${ }^{1}$ With recent improvements in screening techniques and the advent of neonatal screening programmes we expect to detect infants with severe and profound hearing loss within the first year of life and to see a significant number under the age of 6 months. It is widely accepted that the earlier the habilitation of a child with a severe or profound hearing loss starts, the better the outcome is likely to be. Markides has demonstrated better speech intelligibility in children who were fitted with amplification in their first six months of life compared with children fitted after this age. ${ }^{2}$ Selection and use of amplification is a part of this habilitative process.

The first problem in the selection of appropriate amplification lies in obtaining sufficient and accurate information about the child's hearing sensitivity over the essential speech frequency range-that is, $500-4000 \mathrm{~Hz}$. Once the infant reaches the age of 6 months or so and is sitting independently with good head control it is usually possible to obtain reliable information about hearing sensitivity using distraction or visual reinforcement audiometric techniques. In an infant whose developmental problems preclude this form of testing, and in infants under the age of 6 months, electric response audiometry is usually required in order to obtain reliable threshold information. The auditory brainstem response is usually the choice for threshold determination in infants. This gives threshold information about high frequency hearing but not low frequency hearing and this is one of its limitations. ${ }^{3}$ To obtain information about the low frequency hearing it is essential to carry out careful observation of the child's responses to auditory stimuli in the sound field. The initial selection of amplification characteristics and fitting of hearing aids is usually based, of necessity, on rather limited information, particularly in the infant under 6 months of age. The child is monitored closely initially: parents' and teachers' observations are noted and audiological testing of the infant is carried out both with and without hearing aids. In this way a more detailed audiological picture is obtained and changes to the hearing aid prescription made as required.

Amplification is usually recommended for hearing losses greater than $30 \mathrm{~dB}$ averaged across the speech frequency range in the better ear. This, however, is only a guideline, and the individual's needs must be taken into consideration. For example, a child with a lesser degree of hearing loss but with an appreciable speech delay may well benefit from amplification whereas another child may cope adequately with a slightly greater degree of loss. In fitting hearing aids in infants we are usually dealing with more severe losses where amplification is clearly indicated. The difficulties associated with measuring mild losses accurately in infants and in convincing parents that there is a problem usually preclude fitting infants with mild degrees of hearing loss unless there is a clear and demonstrable high frequency hearing loss.

The aim in providing amplification is to make as much speech information as possible available by amplifying speech to fit within the listener's dynamic range of hearing. The dynamic range is the range between the threshold of hearing and the level at which sound becomes uncomfortable-the loudness discomfort level. The more severe the hearing loss, the more limited the amount of speech information that can be made available. Hearing aid selection involves choosing the electroacoustic characteris- tics of the hearing aid (gain, frequency response, and maximum output) in order to achieve the above. The way in which these characteristics should be chosen has been the subject of much research and publication over the years and a number of prescriptive techniques have been put forward. ${ }^{4-6}$

Whatever method of selection is used it is necessary to evaluate the hearing aid fitting and one method is to obtain aided sound field thresholds (using warble tone or narrow band noise stimuli) when the infant is wearing the hearing aids. This gives a measure of the amount of speech frequency information that is available to the child. It is important to avoid excessively high levels of amplification that will prove uncomfortable and lead to the child rejecting the hearing aid, but it is equally important to ensure that enough amplification is being used.

The potential of high levels of sound to damage hearing is well known. Naturally there is concern about the potential of high output levels produced by present day hearing aids to further damage the users residual hearing. This has been the subject of a number of surveys, ${ }^{7}$ but no conclusive evidence has been produced. Any possible risk must be weighed against the disadvantage to the child in not utilising residual hearing to the full.

\section{Hearing aids}

Hearing aids are generally relatively unsophisticated devices, although advanced signal processing systems are being developed. At present most are basically amplifiers with some facility to (i) manipulate the relative amount of amplification at different frequencies, (ii) control the maximum output, and (iii) selectively alter the gain in order to 'compress' a wide range of sounds to fit within the user's dynamic range. A hearing aid basically consists of a microphone that converts the incoming sound to an electrical signal, an amplifier with a variable gain control, and a receiver that converts the amplified electrical signal back into an acoustic signal. The power supply is provided by a battery.

In postaural aids all these components are housed in one case, which is worn behind the ear. The aid is coupled to the earmould, which is individually made for the infant's ear, via a small elbow or hook on the hearing aid. This is connected to the tubing that provides a channel through the earmould and delivers the amplified acoustic signal deep into the meatus of the ear.

In the ear aids, where all the components are mounted in an earmould that fits into the canal and concha of the external ear, are now available. These aids are generally not suitable for growing infants because of the cost of frequent replacement of the system caused by growing ears.

With body worn hearing aids, the microphone, amplifier, and batteries are contained in a unit that is mounted on the child's chest, usually in some sort of harness. The receiver is external and is connected via a lead to the body worn unit. The receiver itself clips directly into the earmould and is held there by a retaining spring clip.

All of these types of aid described so far (postaural, in the ear, and body worn) deliver the acoustic signal via air conduction deep into the external meatus of the ear and the resulting acoustic signal is transmitted via the middle ear to the cochlea and beyond in the normal way. It is also possible to use a bone conduction hearing aid. In this case the 
receiver consists of a head worn vibrator (rather like the bone conductor on an audiometer), which is worn on the mastoid process and delivers an acoustic signal to the cochlea by vibration of the skull and soft tissue. The remaining components may be mounted in either a postaural or a body worn unit. These aids are only used when there is no alternative-in cases of atresia, where the normal air conduction route is not available, or sometimes in cases of chronically discharging ears where the condition is aggravated by the presence of an earmould. This type of aid is difficult to use and retain with young children. Recent developments in the area of implanted bone conduction aids show great promise for children with problems such as bilateral atresia. $^{8}$

For the vast majority of young children the choice is between a postaural or body worn instrument. There is little to choose between the two in terms of technical performance and postaural aids will be the usual option. The only exception to this is children with low frequency residual hearing only (with so called left hand corner audiograms) where body worn aids may be considered. With the range of smaller postaural aids now available it is usually possible to fit the smallest ears. It may be necessary to use minihooks on hearing aids, double sided adhesive discs which secure the aid to the side of the head, special clips attached to the aid, or plastic retaining devices to help maintain the aid in position. The consensus regarding binaural fitting (one aid for each ear) is that this should be done routinely unless there is a good reason not to. There are a number of advantages in binaural as opposed to monaural fitting including enhanced sound localistation ability and the achievement of higher signal levels because of the binaural summation effect. ${ }^{9}$

One of the problems with hearing aid use is the poor signal:noise ratio which occurs when all acoustic stimuli arriving at the microphone of the aid are amplified. One way of overcoming this is by the use of remote microphone systems, for example in the use of radio systems. These are commonly used by hearing impaired children in educational settings and by some preschool children at home. The mother or teacher wears a transmitter that picks up her voice and transmits it by radio frequency to a receiver worn by the child. In this way the problems of distance between speaker and listener and of background noise can be overcome to some extent and a better signal:noise ratio achieved. Evans discusses hearing aid systems more fully. ${ }^{10}$

\section{Ear moulds}

The earmould is an integral part of the amplication system. Its function is to deliver the amplified sound from the hearing aid receiver into the ear canal. It also serves to retain a postaural aid in position behind the ear or the receiver of a body worn hearing aid in the ear. In order to do this an earmould must be manufactured from appropriate material so that it is comfortable to wear for all of a child's waking hours and is capable providing an extremely good acoustic seal. The need for the former is obvious if a child is to accept hearing aids; the latter requirement is particularly important when high levels of gain and output are required as in cases of severe and profound hearing loss. Failure to provide a good acoustic seal results in acoustic 'feedback'-a characteristic high pitched whistle that arises when amplified sound escapes around a poorly fitting earmould and is picked up by the microphone of the hearing aid and reamplified. The better the acoustic seal provided by the earmould the less the likelihood of this type of feedback occurring. There are other causes of feedback that may also need to be considered. ${ }^{11}$

The problems associated with the provision of good ear- moulds for children are well known and have been the subject of a number of surveys. ${ }^{12}$ With a skilled impression taker using a standard technique, however, ${ }^{13}$ and the availability of suitable earmould materials, good earmoulds can be provided. It is essential that earmoulds for children are made of soft materials-for example, soft acrylic, silicone, or molloplast in order to satisfy the requirements of comfort and a good acoustic seal. In the case of rapidly growing infants, who require extremely well fitting earmoulds in order to deliver the required gain levels, it may be necessary to renew earmoulds as frequently as every three or four weeks.

\section{Management of the hearing impaired infant}

This requires a team approach to deal with the needs of the infant and family. In addition to the audiological aspects involved in the measurement of hearing sensitivity and the selection, provision, and monitoring of amplification there are more specifically medical and educational needs. The infant will be seen by a consultant in otolaryngology or audiological medicine to assess whether there is any possible medically or surgically correctable cause of the hearing loss, or part of it, and to authorise the fitting of hearing aids. In addition, the child should be seen for a full developmental assessment including assessment of visual problems and for investigations into the aetiology of the hearing loss with referral for genetic counselling where appropriate. Hearing impaired infants and their families also require educational and rehabilitative support. In most areas infants with a permanent hearing loss and fitted with hearing aids would expect to receive regular home visits from a specialist teacher of the deaf whose role is to provide guidance and support to the family as a whole, rather than having a very specific teaching role, as well as maintaining an overview of the child's progress. Increasingly specialist speech therapists with additional qualifications in working with the hearing impaired are also involved in this area. Close liaison between all these professionals and the family is essential. It is also important to inform families about relevant voluntary organisations that can provide help and support.

SALLY WOOD

BARRY MCCORMICK

Children's Hearing Assessment Centre, General Hospital,

Park Row,

Nottingham NGI $6 \mathrm{HA}$

1 Martin JAM, Bentzen V, Colley JRT, et al. Childhood deafness in the European Community. Scand Audiol 1981;10:165-74.

2 Markides A. Age at fitting of hearing aids and speech intelligibility. $\mathrm{Br} \mathcal{J}$ Audiol 1986;20:165-8

3 Mason SM, McCormick B, Wood SA. Auditory brain stem response in paediatric audiology. Arch Dis Child 1988;63:465-7.

4 Gengel RW, Pascoe D, Shore I. A frequency response procedure for evaluating and selecting hearing aids for severely hearing impaired children. f Speech Hear Disord 1971;36:341-53.

5 Seewald RC, Ross M, Spiro MK. Selecting amplification characteristics for young hearing impaired children. Ear Hear 1985;6:48-53.

6 Byrne D, Dillon H. The National Acoustic Laboratories (NAL) new procedure for selecting the gain and frequency response of a hearing aid. Ear Hear 1986:7:257-65.

7 Markides A, Aryee DTK. The effect of hearing aid use on the user's residual hearing. Scand Audiol 1978;7:19-26.

Hakansson B, Tjellstom A, Rosenhall V, Carlsson P. The bone anchored hearing aid. Acta Otolaryngol (Stockh) 1985;100:229-39.

Wernick IS. Use of hearing aids In: Katz J, ed Handbook of clinical audiology. 3rd Ed. Baltimore: Williams and Wilkins, 1985:911-35.

10 Evans P. Hearing aid systems. In: McCormick B, ed. Paediatric audiology 0-5 Evans P. Hearing aid systems. In: McCormick B, ed. Paediatric a
years. London: Taylor and Francis (Whurr), 1988:265-302.

11 Pollack MC. Electroacoustic characteristics. In: Pollack MC, ed. Amplification for the hearing impaired. 2nd Ed. New York: Grune and Stratton, 1980: for the

12 Nolan M. Earmoulds. In: McCormick B, ed. Pediatric audiology 0-5 years. London: Taylor and Francis (Whurr), 1988:325-47.

13 British Society of Audiology. Recommended procedures for taking an aural impression. Br $\mathcal{J}$ Audiol 1987;20:315-7. 